\title{
Erratum to: Positivity violations of the density operator in the Caldeira-Leggett master equation ${ }^{\star}$
}

\author{
Eur. Phys. J. D (2019) 73: 53, https://doi.org/10.1140/epjd/e2019-90604-4
}

Gábor Homa ${ }^{1}$, József Zsolt Bernád $\mathrm{d}^{2,3, a}$, and László Lisztes ${ }^{4}$

${ }^{1}$ Department of Physics of Complex Systems, Eötvös Loránd University, ELTE, Pázmány Péter sétány 1/A, 1117 Budapest, Hungary

2 Department of Physics, University of Malta, Msida MSD 2080, Malta

${ }^{3}$ Institut für Angewandte Physik, Technische Universität Darmstadt, 64289 Darmstadt, Germany

4 Axioplex Ltd., Külső Szilágyi út 36, 1048 Budapest, Hungary

Received 17 March 2019

Published online 25 June 2019

(c) EDP Sciences / Società Italiana di Fisica / Springer-Verlag GmbH Germany, part of Springer Nature, 2019

This erratum corrects three mistakes. The first is a typo, the second one is an unclear statement about the states used in the numerical simulations, and the third is a misused reference with respect to an equation.

1) The equation before equation (13) should read

$$
\begin{aligned}
1-2 F_{1}(t) & =\langle\hat{x} \hat{p}+\hat{p} \hat{x}\rangle-2\langle\hat{x}\rangle\langle\hat{p}\rangle=2 \sigma_{p x}^{2}, \\
F_{2}(t) & =-4 D_{p p}\left(\left\langle\hat{x}^{2}\right\rangle-\langle\hat{x}\rangle^{2}\right)=-4 D_{p p} \sigma_{x x}^{2} .
\end{aligned}
$$

2) The states

$$
\begin{aligned}
\rho(R, r, 0)= & \frac{H_{n}\left(\beta R+\frac{\beta r}{2}\right) H_{n}\left(\beta R-\frac{\beta r}{2}\right)}{2^{n} n !} \\
& \times \mathrm{e}^{-\frac{1}{2} \beta^{2}\left(2 R^{2}+\frac{1}{2} r^{2}\right)} \sqrt{\frac{\beta^{2}}{\pi}},
\end{aligned}
$$

together with the real parameter $\beta$ being proportional to the inverse width $x_{0}=\sqrt{\hbar /(m \omega)}$ of the quantum harmonic oscillator's ground state describe a general set of states. The eigenstates of the quantum harmonic oscillator are elements of this set and one can obtain them when $\beta^{\prime}=\beta x_{0}=1$. However, the wave packet of each state with $\beta^{\prime}=0.6$ has a larger width than the corresponding eigenstate of the quantum harmonic oscillator and thus the occurrence of the positivity violation is more limited due to the condition

$$
\gamma-2\left(D_{p p} \sigma_{x x}^{2}+2 D_{p x} \sigma_{p x}^{2}\right) \leqslant 0
$$

and the time evolution. In this context, all our figures are produced with less favorable initial states for the demonstration of the positivity violation. In order to show this, we compare in Figure 1 the published Figure 3a $\left(\beta^{\prime}=0.6\right)$ with a figure, where we set $\beta^{\prime}=1$ and the rest of the parameters have the same value as in Figure 3a. In summary, the mistake does not change any of the conclusions of the paper.

3) In the section, where the numerical results are discussed, there are three references to condition (2), which should read condition (13).

* The online version of the original article can be found at https://doi.org/10.1140/epjd/e2019-90604-4.

a e-mail: zsolt. bernad@um.edu.mt 


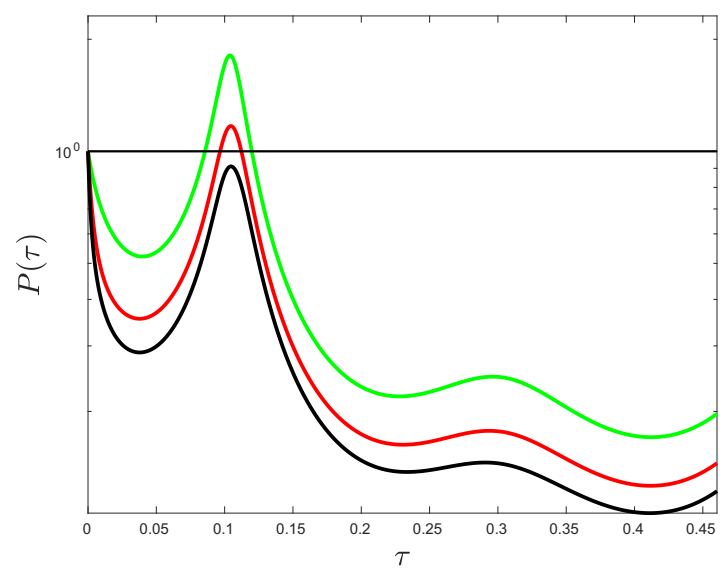

(a)

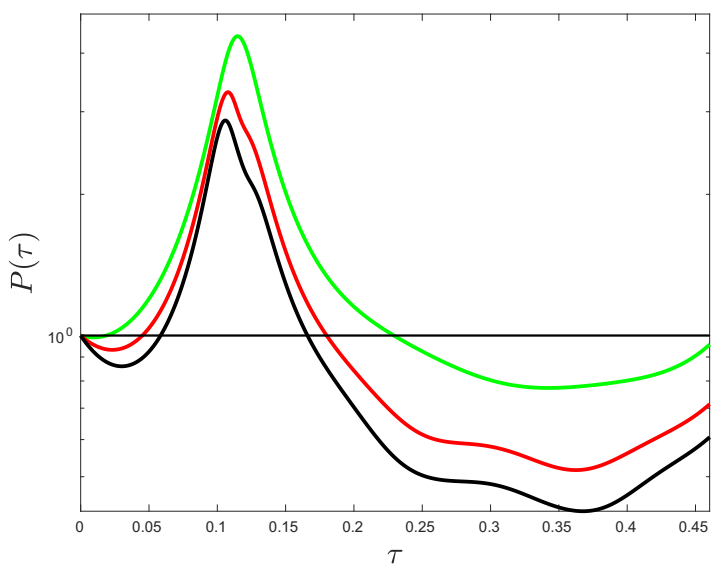

(b)

Fig. 1. Left panel: $\beta^{\prime}=0.6$, Figure $3 \mathrm{a}$ in the publication. Right panel: $\beta^{\prime}=1$, the first three eigenstates of the quantum harmonic oscillator. The three different curves are plotted for the following initial states of equation (1): $n=0$ (green); $n=1$ (red); and $n=2$ (black). 